\title{
Bearing Grounding Mechanical Properties of Engineering Vehicle Retreaded Tire
}

\author{
Qiang Wang ${ }^{1 *}$, Li Jiang ${ }^{1}$ \\ ${ }^{1}$ School of Automotive and Transportation Engineering, Heilongjiang Institute of Technology, Harbin 150050, China
}

\begin{abstract}
In this study, a multivariate composite layer model, computer geometry models, contact pair models, finite element analysis (FEA) models, and bearing grounding mechanical property test system were constructed. FEA and experimental study on the bearing grounding mechanical properties of retreaded tires of engineering vehicles were processed. Therefore, features and rules of load grounding pressure, load grounding mark, load grounding area, load grounding coefficient, and load grounding hardness coefficient of retreaded tires under the static grounding working condition were summarized, from which load-bearing grounding mathematical models of $26.5 \mathrm{R} 25$ engineering vehicle retreaded tire were constructed. Analysis results show that the grounding pressure at the tread center of grounding pressure and grounding friction, which increased along the tire width and rolling direction at different degrees, were the smallest under the static grounding working condition. The shape of the earthing marks turned from circular to elliptical, and then close to the rectangle with the load increase, and finally into an approximate saddle shape. When the tire pressure was certain, the earthing area increased gradually with the increasing load and the increasing trend was nonlinear. The ground pressure and grounding force on the tire shoulder of the engineering retreaded tire was the largest, which could be damaged easily.
\end{abstract}

\section{Introduction}

In recent years, mining, construction, and other industries have developed rapidly. Thus, the demand for engineering vehicle tires has increased on a daily basis. Engineering vehicle tires are typically used in open-pit mining areas, such as those of soil and stone, which are loaded with large, frequent starting and braking, and are affected by bulge; thus, the production rate of waste tires is rapid and the production yield is large (Junxiong, 2016; Daochun, 2016; Jun 2014). The amount of rubber used for an engineering vehicle tire is approximately $15 \%$ of the total rubber consumption of the tire manufacturing industry. Therefore, increasing the renovation rate of waste engineering vehicle tires can effectively improve the utilization rate of such tires, thereby saving rubber resources and promoting green environmental protection. Furthermore, pollution caused by waste tires would be turned into energy. At present, whether in China or in developed countries such as the United States, Japan, and South Korea, most studies focus on tire refurbished industry state, related policy analysis, technology equipment system development of the loading vehicle tire renovation, loading vehicle tire renovation technology, product quality evaluation of the loading vehicle tire renovation, tire tread modification, enhancement technology of the loading vehicle, and other factors (European Rubber Journal GROUP, 2013; Rubber World Group, 2013; Indian/international rubber journal group,
2013). Numerous studies on vehicle tires and retreaded tires have been conducted in those developed countries and in China. However, limited studies have focused on the macro and micro mechanical performance of engineering vehicle retreaded tires. Apart from the results presented by the researchers, published studies are rarely found in recent years. Owing to the lack of basic technology for refurbished engineering tires, tire tread is not wear-resistant during usage, and has other damage forms; for example, it is easy to crash cost block and blast on its appearance, thereby seriously affecting the popularization and application of engineering retreaded tires. To this end, this paper qualitatively and quantitatively described and evaluated the grounding contact pressure characteristics of engineering vehicle retreaded tires by building the computer geometric model, FEA model, and grounding contact pressure characteristic test system. Results provide important theoretical guidance for the study on the performance evaluation and optimization of the renovation technology for engineering vehicle retreaded tires.

\section{FEA model of engineering vehicle re- treaded tire}

The finite element model constructed by ANSYS Workbench software is shown in Fig. 1. The model consists of 19,976 degrees of freedom; 68,377 nodes; and 41,554 units. The pair model construction of contact with the 
ground is shown in Fig. 2. The contact friction coefficient between rigid ground and flexible tire was set to be 0.9 (Fenglin, 2016; Shan and Wei, 2016). The material parameters, such as tread, buffer rubber, tire side, toe mouth rubber, and steel wire ring were measured, as shown in Table 1. The material parameters of the old tire body and belt layers were measured by experiment, as shown in Table 2. Mooney-Rivlin model was applied to simulate the tread, buffer rubber, tire side, and toe mouth rubber. The solid unit was used to simulate the steel wire ring, while the old tire body and belt layers were simulated using a layer unit.

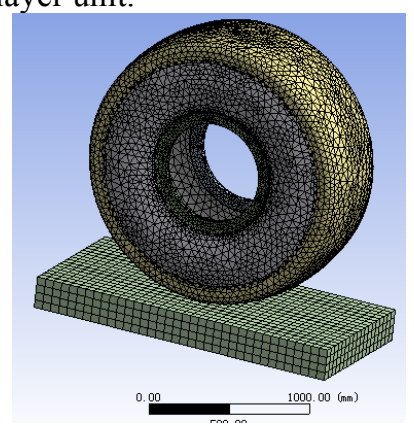

Fig. 1Finite model

Table 1 Material parameters of tread

\begin{tabular}{lccc}
\hline Material & Elasticity modulus $(\mathrm{MPa})$ & Poisson's ratio & Density $\left(\mathrm{kg} / \mathrm{m}^{3}\right)$ \\
\hline Tread & 7.26 & 0.48 & 1790 \\
\hline Buffer rubber & 5.94 & 0.48 & 1020 \\
\hline Tire side & 10.36 & 0.48 & 1240 \\
\hline Toe mouth rubber & 12.14 & 0.48 & 1370 \\
\hline Steel wire ring & $2.12 \mathrm{e} 5$ & 0.29 & 7850 \\
\hline
\end{tabular}

Table 2 Material parameters of old tire body and belt layer

\begin{tabular}{|c|c|c|c|c|c|c|c|c|c|c|}
\hline \multirow{2}{*}{ Material } & \multicolumn{3}{|c|}{ Elasticity modulus (MPa) } & \multicolumn{3}{|c|}{ Shear modulus (MPa) } & \multicolumn{3}{|c|}{ Poisson's ratio } & \multirow{2}{*}{$\begin{array}{c}\text { Density } \\
\mathrm{kg} / \mathrm{m}^{3}\end{array}$} \\
\hline & $E_{1}$ & $E_{2}$ & $E_{3}$ & $G_{12}$ & $G_{23}$ & $G_{31}$ & $\mu_{12}$ & $\mu_{23}$ & $\mu_{31}$ & \\
\hline $\begin{array}{c}\text { Old tire } \\
\text { body }\end{array}$ & $9.7 \mathrm{e} 4$ & 6.96 & 6.96 & 6.04 & 3.26 & 3.26 & 0.398 & 0.398 & 0.48 & 4580 \\
\hline $\begin{array}{c}\text { Belt } \\
\text { layer }\end{array}$ & $1.58 \mathrm{e} 5$ & 2.62 & 2.62 & 9.88 & 2.58 & 2.58 & 0.344 & 0.344 & 0.48 & 6480 \\
\hline
\end{tabular}

\section{Finite element numerical simulation of grounding mechanical properties of en- gineering vehicle retreaded tir}

\subsection{Analysis of grounding pressure characteris- tic}

\subsubsection{FEA of grounding pressure distribution}

The grounding pressure distribution cloud figure of engineering retreaded tire under the tire pressure of 600 $\mathrm{kPa}$ and load of $135 \mathrm{kN}$ is shown in Fig. 3. The cloud image of grounding friction stress distribution is shown in Fig. 4 Figure 3 shows that the pressure distribution of retreaded tires in the grounding area is uneven. The grounding pressure of tire shoulder is $10.503 \mathrm{MPa}$, while the minimum value is $1.167 \mathrm{MPa}$ in the grounding center area. The minimum value of the pressure increases at a different degree from the grounding center along the width of the tire and the circular rolling direction. Figure 4 shows that the friction distribution in the grounding area of the retreaded tire is not evenly distributed. The contact friction on the shoulder position of the tire reaches the maximum at $8.51 \mathrm{MPa}$; the friction value of the grounding center area reaches the minimum at $1.94 \mathrm{MPa}$, and then the friction value increases from the grounding center along the tire rolling and width directions in a different degree. 


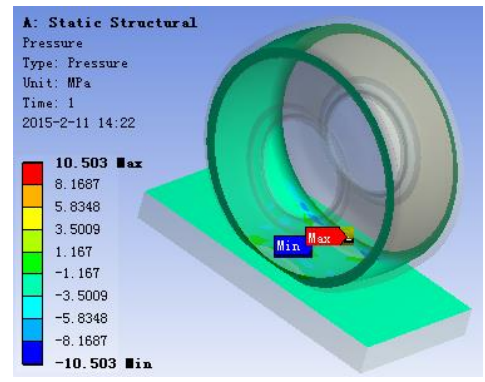

Fig. 3 Cloud of grounding pressure distribution

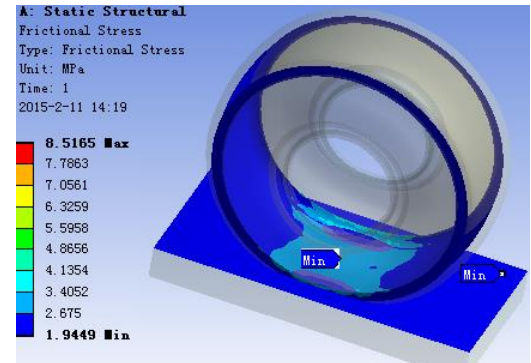

Fig. 4 Cloud of grounding friction stress distribution

\subsubsection{Test of grounding pressure distribution}

The grounding pressure distribution of $26.5 \mathrm{R} 22.5$ engineering retreaded tires together with the new tires of the same brand and the same model were tested under the following working conditions: one with constant tire pressure and changeable load, and the other with constant load and changeable tire pressure. In this manner, the grounding pressure distribution rules of engineering retreaded tires were studied and compared with those of the new tire and simulation value.

The composition of the grounding pressure test sys- tem is shown in Fig.5. The test system installation is shown in Fig. 6. The engineering retreaded tire under the test marked as 4 in Fig. 13 is fixed on the rotation axis marked as 3, and the air compressor marked as 1 is treated with the tire marked as 4 . In addition, the tire pressure is calibrated by the tire pressure gauge marked as 5 in Fig. 13, and the force loader marked as 6 moves up and down to implement loading and unloading. Moreover, the loading plate marked as 8 in Fig. 13 simulates the road surface, and the diaphragm-type pressure sensor marked as 9 is distributed in the contact area of the tire tread and ground.

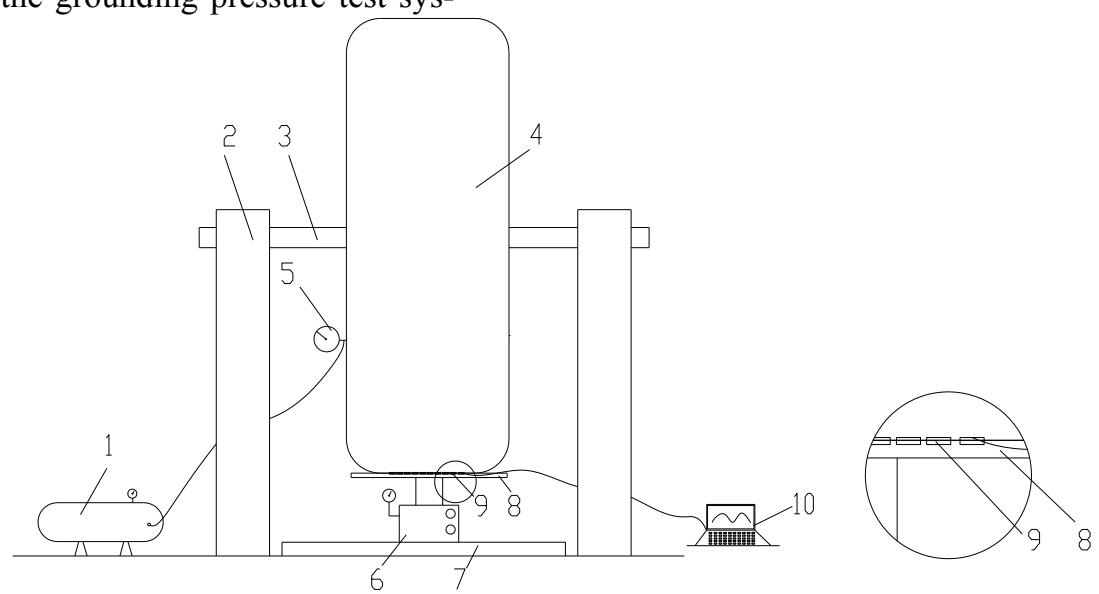

1- Air compressor 2- Support 3- Rotation axle 4- Tire under test 5- Tire pressure gauge 6- Force loader with digital display 7- Platform 8- Loading plate 9- Diaphragm-type pressure sensor 10- Data indicator

Fig. 5 Composition of test system 


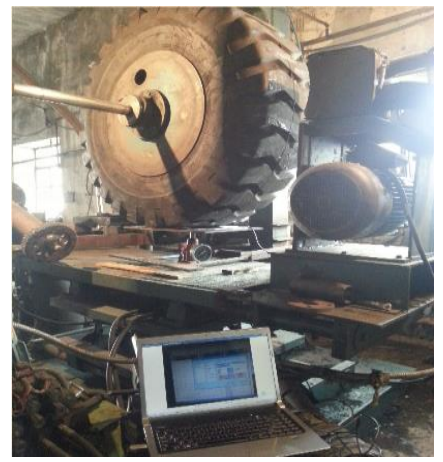

Fig.6 Test system installation

\subsubsection{Analysis of grounding pressure results}

According to the simulation and test results, the grounding pressure distribution of the engineering retreaded tires approximately turned out to be a "V" shape, and minimum pressure value was in the tire grounding center area. However, the value increased gradually along the direction of the width and rolling circumference, in which the trend of the increase along the tire width direction was nonlinear, the trend of increase along the direction of the rolling circumference was approximately linear, and the maximum could be reached at the tire shoulder and grounding edge along the rolling direction. The results show that the part of the tire shoulder is more likely to be damaged than other parts of the engineering retreaded tire during usage. When the tire pressure was certain, the grounding pressure along the tire width direction and rolling circumference direction increased as the load increased; when the load was certain, the grounding pressure remained similar under the two types of working condition, of which the tire pressure was $\mathrm{P}=$

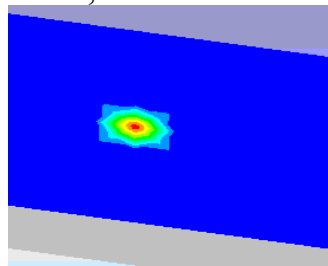

(a) Load $115 \mathrm{kN}$

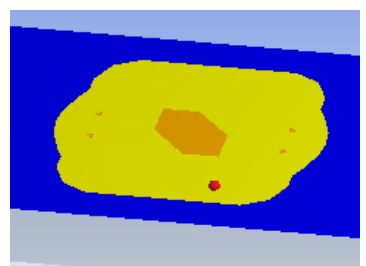

(b) Load $155 \mathrm{kN}$
$450 \mathrm{kPa}$ and $\mathrm{P}=500 \mathrm{kPa}$. When the tire pressure continued to increase, the grounding pressure gradually increased. The results show that the contact pressure of the shoulder position of the engineering retreaded tire was greater, and the rubber on the tire shoulder position was thicker and arc-shaped, which easily formed the stress concentration. Therefore, the tire shoulder position easily occurred in the form of the tearing failure of dissection from the retreaded tire tread.

\subsection{Characteristic analysis of grounding mark and grounding area}

\subsubsection{Characteristic analysis of grounding mark and side slipping state}

When the tire pressure was $600 \mathrm{kPa}$ and the vertical loads were 115,155 , and $185 \mathrm{kN}$, the grounding mark shape of engineering retreaded tire is shown in Fig. 7, and the cloud of tire sliding distance is shown in Fig. 16.

Fig. 7 Grounding mark shapes of tire

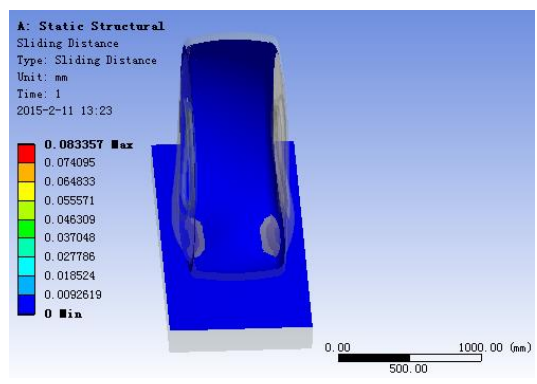

Fig.8 Cloud of tire sliding distance 
Fig.7 shows that the grounding mark of the engineering retreaded tire is symmetric geometry, but with the increase of the load, the area of the grounding mark gradually increases, while the shape turns to oval from the original round, and then gradually turns to rectangle. When the load is large, the distribution along the tire circumference direction is in a saddle shape. As shown in Fig. 8, the retreaded tire produces a smaller side-slipping amount along the axial direction, which is $0.08 \mathrm{~mm}$; thus, this condition is ignored in the actual work, which is consistent with the actual situation.

\section{Conclusion}

(1) Under static grounding working condition, the grounding pressure and grounding friction of the tread center point around the grounding area of the engineering retreaded tire were the smallest; they increased in different degrees along the direction of the tire width and rolling directions.

(2) The landmark shape of the retread tires varies with the increase of load from round to oval, and then near the rectangle, finally resembling a saddle shape.

(3) When the tire pressure is constant, the ground area of the retread tire increases with the increase of load and shows a nonlinear trend.

\section{Funding}

This study was supported by Basic Scientific Research Operating Expense Funding Project of Provincial Univeristies in Heilongjiang Province (2018CX07); Natural Science Fund Project in Heilongjiang Province (LH2019E115); Heilongjiang Institute of Engineering Ph. D. Fund. (2016BJ02).

\section{References}

1. European Rubber Journal GROUP. Bridgestone opens tire retreaded plant in Osaka[J]. European Rubber Journal, , Vol. 195, No. 5 (2013), pp. 6574.

2. Fan Ningning, $\mathrm{Mu}$ Shouyong. The Design of 12.00R20 18PR Reinforced All-Steel Loading Radial Tire $[\mathrm{J}]$. Rubber Technology, Vol. 6 (2013), pp. 44-47.

3. Indian/international rubber journal group. Michelin debuts off-road XDY-EX Pre-Mold retreaded Tire [J].Indian/international rubber journal, Vol. 158 (2013), pp. 345-358.

4. Jiang Fenglin. The Finite Element Analysis is Applied to the Optimization of the Structure of 3700r57 Massive Engineering Mechanical Meridian Tires [J]. Tire Industry, No. 03 (2016), pp. 147-149.

5. Jing Yu, Jiang Zhanghua, Tan Lin.11.00R2018PR the Design of the All-Steel Lightweight Load Radial Tire [J]. Rubber Technology, Vol. 12
(2016), pp. 37-39.

6. Li Renguo, Zhang Peng, Cheng Gang, and so on. The Design of 14.00R20 20PR Full Steel Loading Radial Tire[J].Rubber Technology, Vol. 33, No. 6 (2013), pp. 342-344.

7. liu Daochun. The retreaded tire Industry Leads the Future of Low-Carbon Energy Saving [J]. Modern Rubber Technology, No. 02 (2016), pp. 9-14.

8. Liu Shenglin. The Design of the 385/55R19.5 Wide-Base, No-Enner Tube and All- Steel Load Radial Tire [J].Tire Industry, No. 09 (2016), pp. 529-532.

9. Liu Ye, Lian Zheman. Finite Element Analysis of Automobile Tire Based on ANSYS [J]. Mechanical Engineer, No. 03 (2014), pp. 105-106.

10. Rubber World Group. U.S. Retreaded tire market increased 35 percent[J]. Rubber World: The Technical Service Magazine for The Rubber Industry, Vol. 247, No. 5 (2013), pp. 136-149.

11. Wang Jun, Li Liang, Sun Lin, Sha Changxin. Finite Element Analysis of 245/70R16 Tire [J]. Tire Industry, No. 09 (2016), pp. 520-528.

12. Wang Qiang, Qi Yingjie. Construction of MultiComposite Layer and Mechanical Model of the Engineering retreaded tire [J]. Journal of Zhengzhou University ( Engineering Science) , 2015,36 (1) : 87-90.

13. Wei Yintao, Li Yong, Feng Xijin, and so on. Tire Theory and Technology [M]. Beijing : Tsinghua University Press (2013), pp. 128-142.

14. Yan Shan, Wang Wei. Finite Element Analysis and Experimental Study on Complex Tread Pattern Tire[J].Rubber Industry, No. 02 (2016), pp. $102-106$

15. Zhong Junxiong. Analysis on the Necessity of retreaded tire Product Quality Monitoring [J]. New Technology and New Product in China, No. 11 (2016), p. 161.

16. Zhu Jun. Study on the Resource Recovery Model of Waste Tires Based on the System Theory [J]. Master's thesis of Dongbei University, No. 06 (2014), pp. 2-9 